\title{
Descending necrotizing mediastinitis due to odontogenic infection: an unusual case report
}

\section{Introduction}

Descending Necrotizing Mediastinitis (DNM) is a deep thoracic infection that affects the mediastinum (pericardial space located within the thoracic region) that comes primarily from untreated odontogenic or oropharyngeal infections. The posterior mediastinum is an anatomical region that can be reached by these infectious proliferations due to the proximity of the oropharyngeal area with the retropharyngeal and parapharyngeal spaces. ${ }^{1}$ DNM may initiate clinical alterations such as: edema in the submandibular region conferring "bull's neck" aspect, trismus, cervical extension limitation, redness, fever, dysphasia, dyspnea besides pleural and pericardial effusion. ${ }^{1,2}$ The history of recent tooth extractions and odontogenic infections is common since dental infections usually present a wide microbiological variety. ${ }^{3}$ Regarding the risk of death, DNM leads a high morbidity to the patient, considering the presence of septic shock between $40-50 \%{ }^{1}$ of the cases. This severe and aggressive behavior explains the importance of complementary examinations for diagnostic elucidation such as chest and cervical Computed Tomography (CT), complete blood count, blood culture and antibiogram. ${ }^{4}$ The standardized treatment for deep cervical and thoracic infections, such as mediastinitis and cervical fasciitis, is to maintain free and functional airways through end tracheal intubation, cricothyroidectomy, or tracheostomy as well as drainage of congestive fascial spaces and removal of the etiological factor for maintenance of the individual, associated with antibiotic therapy intravenously for the time needed to ensure patient's functionality. The aim of this paper is to report the case of a young male patient affected by an odontogenic infection with severe systemic complications.

\section{Case report}

A 21-year-old normossemic patient sought care in an emergency hospital with a major complaint of neck swelling and pain to breathe, talk, eat and drink water, presenting anodontogenic infection of the lower right first molar tooth that evolved for 9 days with no previous medical assistance. The clinical aspect showed (Figure 1) redness and extensive swelling of hardened consistency of neck, submandibular, submental and sublingual spaces bilaterally, trismus, dysphasia, dyspnea, dehydration, speech alteration and limited cervical mobilization. The diagnostic hypothesis was deep cervical infection and descending mediastinitis arising from Ludwig's Angina at the expense of a dental infection source. Due to the clinical characteristics presented, complementary exams were requested for diagnostic assistance: chest, neck and face CTs, complete blood count, hemoculture, antibiogram, renal function markers and rapid HIV test (as the request of the patient).

The initial thorax CT (Figure 2) evidenced a considerable increase in the mediastinal planes, with inflammatory areas in the upper and anterior compartments, pleural and pericardial effusion with mediastinal lymph nodes with altered and reactional appearance. The initial cervical CT (Figure 3) showed multiple fluid collections with gaseous foci in the left, parapharyngeal, anterior cervical, right lateral, retropharyngeal and submandibular spaces, with

\author{
Volume 4 Issue 6 - 2017
}

Breno Souza Benevides' Hermes Melo
Teixeira Batista ${ }^{2}$ Mariana Canuto Melo de
Sousa Lopes ${ }^{3}$ Josfran da Silva Ferreira Filho
Antonio Wisdamy Pinto Saraiva ${ }^{4}$ Fabrício
Bitu Sousa $a^{5}$ Maria Elisa Quezado Lima Verde
and Bruno Frota Amora Silva

'Oral and Maxillofacial Surgeon, Dentistry College, Brazil

${ }^{2}$ Anesthesiologist, Cariri Regional Hospital, Brazil

${ }^{3}$ Oral Medicine and Oral Pathology, Federal University of Ceará, Brazil

${ }^{4}$ Thoracic surgeon, Cariri Regional Hospital, Brazil

${ }^{5}$ Oral Medicine and Oral Pathology, Federal University of Ceará, Brazil

${ }^{6}$ Oral and Maxillofacial Surgeon, Dentistry College-Unichristus, Brazil

Correspondence: Hermes Melo Teixeira Batista, Anesthesiologist. MSc Medical Science. Cariri Regional Hospital, Brazil,Email hermesmelo@oi.com.br

Received: March 29, 2017 | Published: October 04, 2017

communication between them and a determined extensive mucosal bulging and reduction of the oropharyngeal space. Densification of the muscular planes and glottic region with extensive edema in the visceral space were visualized, characterizing a diffuse and extensive inflammatory process in the cervical region. The patient did not present seropositivity for the HIV virus, exhibited laboratorial values compatible with normal renal function, blood current mixed microbiota with the predominance of Staphyloccuscapitis and sensitivity to all the antibiotics tested, ruling out the presence of super-resistant bacteria. The initial hemogram showed an increased marked number of leukocytes and neutrophils, suggesting a recently established infectious and inflammatory condition. As soon as the diagnostic had been confirmed it was opted for early surgical intervention. The procedures consisted of thoracotomy with the objective of managing pericardial and pleural effusions (Figure 4) to avoid the patient's septic shock; surgical drainage of the right cervical, sublingual, left submandibular, left buccal spaces was also performed plus the extraction of the compromised tooth (Figure 5), indicated as the etiological factor. No airway management surgical procedure was performed. For complete drainage, Penrose and chest drains were installed, which were maintained for 3 and 7 days, respectively. The patient remained sedated for 36 hours postoperatively with or tracheal intubation. The antibiotic therapy of choice was Sodium Piperacillin associated with Tazobactam, $12 \mathrm{~g}$ and $1.5 \mathrm{~g}$ per day, respectively. The patient remained under intensive care for 21 days, for postoperative clinical and imaging follow-up control, as well as the remission of signs and symptoms associated with the systemic condition. Currently, the patient is at 6months of follow-up, in which a satisfactory clinical condition and absence of sequelae are observed. 


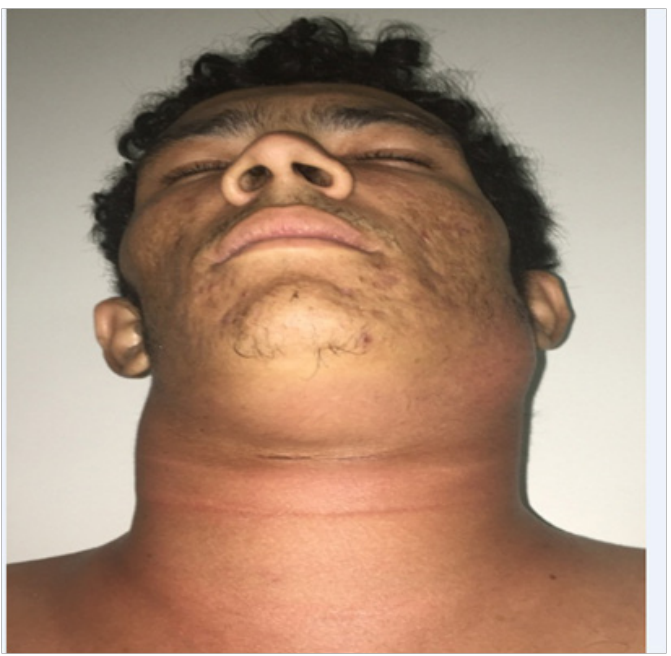

Figure I Clinical appearance evidencing cervical, submandibular, submental and sublingual redness and extensive swelling.
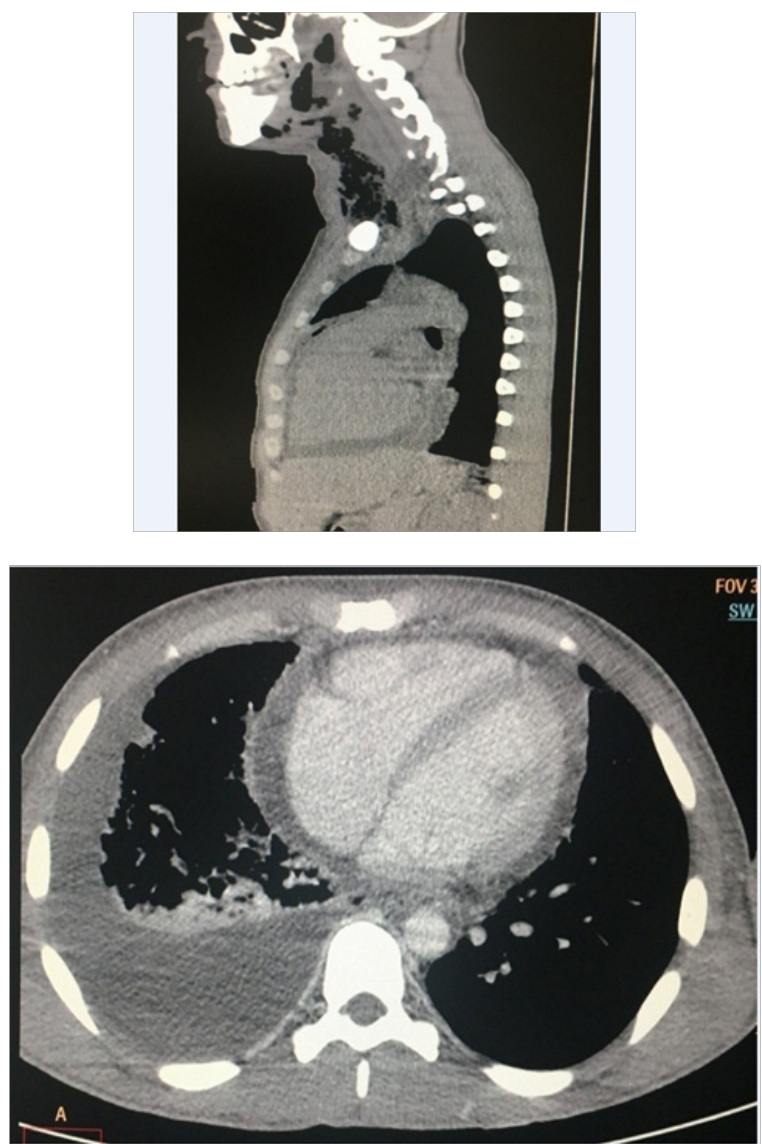

Figure 2 \& 3 CT scans exhibiting liquid collections and extensive cervical and fascial spaces gaseous foci. There are also right interpleural space and pericardial effusion in addition of pulmonary volume reduction of the right lower lobe with focal consolidations and ground glass attenuations.

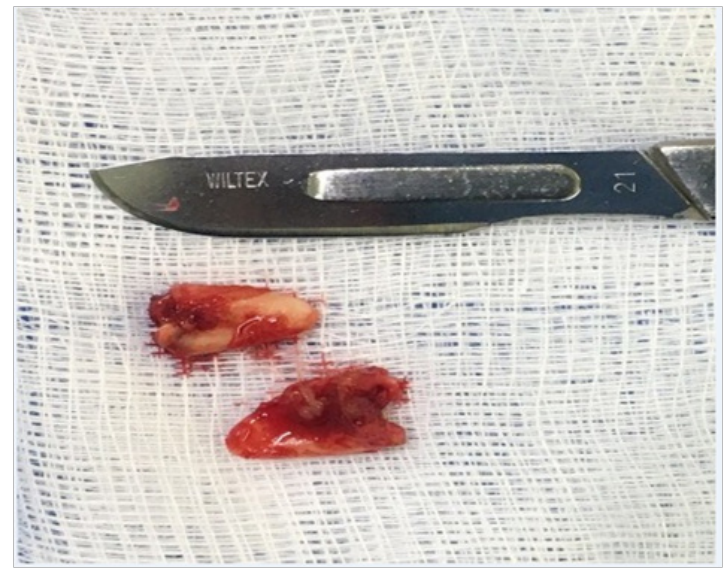

Figure 4 Right anterior lateral thoracotomy for drainage of pericardial and pleural effusion. It is noticed the presence of whitish crust on the right lung surface.

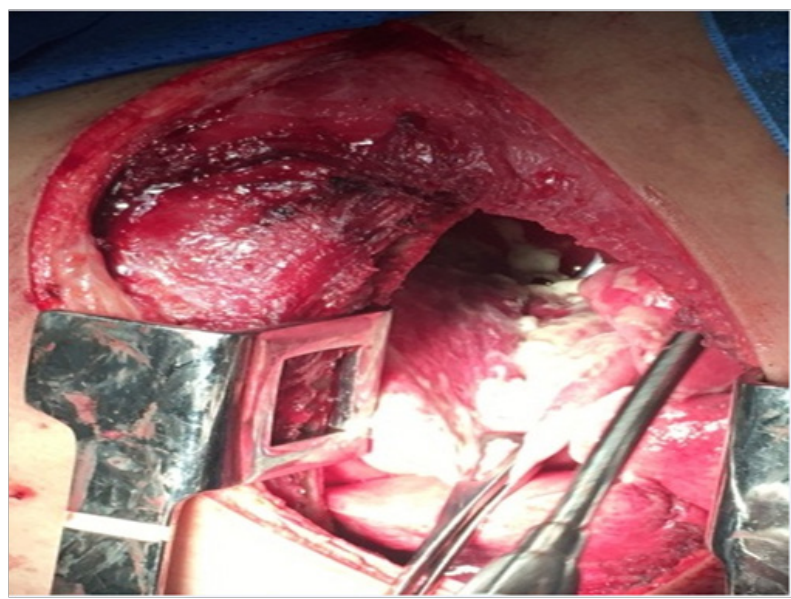

Figure 5 Right lower first molar tooth extracted for elimination of infectious focus; there was an extensive and deep carious lesion enough to damage the dental crown and reduce the dental structure only to the residual roots.

\section{Discussion}

Mediastinitis is an uncommon and low-incidence complication when compared to other situations such as septic shock, pneumonia and airway obstruction with indication of tracheostomy. ${ }^{4}$ It is important to emphasize odontogenic infection as an etiological factor and its interaction with cervico-facial spaces. The mortality rate for Necrotizing Fasciitis ranges from $7 \%$ to $20 \% ;{ }^{1}$ however, when there is an association with DNM, the mortality rate increases to $41 \%$, with a high probability that the patient will die if there is a delay in diagnosis and improper drainage. In the presented case report, the patient required early surgical intervention due to the imminent risk of septic shock. DNM can be classified as an acute or chronic process, with the acute type being more aggressive and symptomatic. It is important to start the diagnostic investigation by the physical evaluation associated with vital signs accompanied by thoracic-cervical imaging and laboratorial tests such as complete blood count and blood culture., $\mathrm{CT}$ scans can demonstrate increases in cardiac and mediastinal boss ${ }^{6,7}$ as well as 
evidence of lung collections characteristic of pleural effusion and pericardial effusion, when circumscribed to the cardiac area. These findings are present in the described case report, and the net collection present in the interpleural space evidenced by chest $\mathrm{CT}$ resulted in approximately $1000 \mathrm{~mL}$ in the right thorax. Corroborating current literature, ${ }^{1,4,8,9}$ cases of Mediastinitis arise mainly when odontogenic infection affects more than one cervical space and submandibular space. In the present case, the composite feature of the involved cervical spaces is evident, since the right cervical, sublingual, left buccal and left submandibular spaces were drained simultaneously with the extraction of the right lower first molar compromised tooth. The presence of unilateral pleural and pericardial effusion was also evidenced, for which the surgical access for anterolateral thoracotomy was performed. This is the most recommended surgical approach for deep surgical treatment in cases of $\mathrm{DNM}^{2,5}$ although there are other options such as bithoracotomy and median sternotomy, which present the disadvantage of high risk of suture dehiscence and osteomyelitis., ${ }^{9,10}$ The clinical-surgical therapy should include maintenance of the airways, elimination of the etiological factor, drainage of the deep cervical/thoracic spaces and broad-spectrum antibiotic therapy. ${ }^{1,8}$ Regarding the interdisciplinary conduct of the presented case report, the patient did not present critical condition as to respiratory activity, reason why no airway management surgical procedure was performed. It was opted for drainage of the fascial spaces affected, thoracotomy for pericardial drainage and debridement of the right thorax and mediastinum, as well as the extraction of the compromised tooth that caused the infectious process. Empirical antibiotic therapy was established immediately after clinical examination and continued for all the hospital stay period. Odontogenic infections, along with tonsillar infections, are among the main predisposing factors for the development of Ludwig's Angina. ${ }^{4}$ Infections in posterior lower teeth are associated in greater number to this condition due to their straight drainage to the submandibular space, which is located near the retropharyngeal and lateral pharyngeal spaces. ${ }^{8,10}$ Piperacillinin combination with Tazobactam can be used as first-line therapy to DNM treatment in view of the polymicrobial characteristic of the infection and the broad spectrum of action of these drugs. ${ }^{6}$ In the presented case report, Sodium Piperacillin associated to Tazobactam demonstrated a satisfactory result, culminating in total Remission of Infection.

\section{Conclusion}

DNM is a serious infectious condition of aggressive and proliferative behavior, presenting as main comorbidities the high risk of septic shock, severe airway obstruction, renal failure and death. Face to the clinical and complementary data of the case reported, interdisciplinary conduct and early surgical management of the patient in addition to the supportive antibiotic therapy and intensive medical care were determinant for the success of the treatment and restoration of the Patient's Functional Condition.

\section{Acknowledgements}

None.

\section{Conflict of Interest}

On behalf of all authors, the corresponding author states that there is no conflict of interest.

\section{Compliance with medical standards}

\section{Informed consent}

Written informed consent for publication of the clinical details and images was obtained from the patient.

\section{References}

1. Sarna T, Sengupta T, Miloro M, et al. Cervical necrotizing fasciitis with descending mediastinitis: Literature review and case report. $J$ Oral Maxillofac Surg. 2012;70(6):1342-1350.

2. Souza VC De, Freire ANM, Tavares Neto J. Mediastinite pós-esternotomia longitudinal para cirurgia cardíaca: 10 anos de análise. Rev Bras Cir Cardiovasc. 2002;17(3):266-270

3. Cariati P, Cabello Serrano A, Monsalve Iglesias F, et al. Meningitis and subdural empyema as complication of pterygomandibular space abscess upon tooth extraction. J Clin Exp Dent. 2016;8(4):e469-472.

4. Brito TP, Hazboun IM, Fernandes FL, et al. Deep neck abscesses: Study of 101 cases. Braz J Otorhinolaryngol. 2015;83(3):341-348.

5. Brommelstroet M, Rosa JFT Da, Boscardim PCB, et al. Mediastinite descendente necrosante pós-angina de Ludwig. $J$ Pneumol. 2001;27(5):269-271.

6. Palma DM, Giuliano S, Cracchiolo AN, et al. Clinical features and outcome of patients with descending necrotizing mediastinitis: prospective analysis of 34 cases. Infection. 2016;44(1):77-84.

7. Zanini FD, Stefani E, Santos JC dos, et al. Angina de Ludwig: relato de caso e revisão do manejo terapêutico. Arq Catarinenses Med. 2003;32(4):21-23.

8. Roberson JB, Harper JL, Jauch EC. Mortality associated with cervicofacial necrotizing fasciitis. Oral Surg Oral Med Oral Pathol Oral Radiol Endod. 1996;82(3):264-267.

9. Brotfain E, Koyfman L, Saidel Odes L, et al. Deep neck infection and descending mediastinitis as a complication of propionibacterium acnes odontogenic infection. Case Rep Infect Dis. 2015;130-134.

10. Cai XY, Zhang WJ, Zhang ZY, et al. Cervical infection with descending mediastinitis: A review of six cases. Int J Oral Maxillofac Surg. 2006;35(11):1021-1025. 